\title{
Prevalence of Staphylococcus aureus and antibiotic resistant Staphylococcus aureus in public transport in Bratislava, Slovakia
}

\author{
Alžbeta Medved'ová, Romana Györiová \\ Department of Nutrition and Food Quality Assessment, \\ Faculty of Chemical and Food Technology, Slovak University of Technology in Bratislava, \\ Radlinského 9, Bratislava 812 37, Slovakia \\ alzbeta.medvedova@stuba.sk
}

\begin{abstract}
There is evidence that the transmission of Staphylococcus aureus and methicillin-resistant S. aureus between the community and environmental surfaces still exists. Even the means of this transmission remain uncertain, the public transport system may serve as a potential source of different bacteria, and the contact with contaminated public surfaces may increase the risk for bacterial diseases emergence. This study aimed to investigate $S$. aureus contamination on Bratislava's public transport vehicles. Forty samples of hand-touched surfaces were collected during December 2015 and March 2017 by using surface sampling method. S. aureus was detected in all analysed swabs. Simultaneously, antibiotic resistance of $S$. aureus from swabs was evaluated. Of 40 samples, only $23 \%$ did not contain $S$. aureus resistant to some of 10 analysed antibiotics. On the other hand, the severe prevalence of highly resistant $S$. aureus to penicillin, methicillin, ampicillin, and cefoxitin was confirmed. $15 \%$ of isolates displayed resistance to at least three antimicrobial classes. The amount of $S$. aureus was not significantly influenced by the lines or by the analysed surface (grabs rails or on-board stop buttons). However, there was a statistically significant effect of year period, both between samples from December and March and between samples from the same month but different year. The study confirmed the widespread occurrence of resistant $S$. aureus in public transport vehicles in Bratislava, Slovakia.
\end{abstract}

Keywords: antibiotic resistance, Bratislava, environmental contamination, public transport, Staphylococcus aureus

\section{Introduction}

Public vehicles, among other public areas, are places with easy transfer of bacteria from individual to individual, and connecting people with different social, ethnic, hygienic and health conditions. The bacteria transmission may be unsettling if pathogenic and resistant bacteria occur. While some bacteria are mainly nosocomial, some are also community-acquired. These include coagulasepositive staphylococci and Staphylococcus aureus, which frequently colonize the nasal vestibule and the skin (ECDC, 2017) of about $10-50 \%$ asymptomatic human. However, S. aureus disposes of a remarkably wide range of virulent factors leading to smooth and skeletal muscle paralysis, blood vessels damage, the formation of extensive skin lesions and finally affecting the central nervous system (Arbuthnott et al., 1990).

Moreover, pathogenic $S$. aureus is regarded as a "superbug", due to its amazing capacity to be resistant to a wide range of antibiotics. Strains resistant to methicillin (MRSA), vancomycin (VISA/ VRSA) and many other antibiotics represent an urgent global problem in community as well as in hospital-acquired infections. The mortality of $S$. aureus bacteraemia remains approximately $20-40 \%$ despite the availability of effective anti- microbials (Mylotte et al., 1987; ECDC, 2017). Most hospital-acquired MRSA in Europe belongs to only five clonal lineages, which have distinct geographical patterns of occurrence, whereas the background populations of methicillin-susceptible $S$. aureus (MSSA) are highly diverse, consisting of many lineages that have been widely disseminated due to leisure travel and migration flows (Gaymard et al., 2016).

In the community, $S$. aureus can be quickly transferred from carriers or infected persons via direct contact (Stepanović et al., 2008). It has also been shown that they can survive under dry conditions and can persist on different surfaces for a very long time (Bremer et al., 2004). Moreover, it is well known for its ability to stick to plastic surfaces. Teichoic acids are essential compounds present in the cell wall and are important for adhesion on plastic surfaces thanks to its interaction with other surface polymers (Reffuveille et al., 2017). In this connection, public vehicles may allow its transmission due to the high number of hand-touched surfaces and their permanent contact with transport users (Gaymard et al., 2016).

Bratislava is the capital and the biggest city in the Slovak Republic. Annually, the city public transport provides transfer for approximately 250.000 people in approximately 850 vehicles 
(buses, trams, and trolleybuses). These may serve as a critical reservoir of staphylococci, due to their ability to colonize human skin and also environmental surfaces. In this connection, we decided to describe the prevalence of $S$. aureus and its antibiotic resistance profile on hand-touched surfaces in vehicles moving around the capital city of Slovakia.

\section{Materials and methods}

\section{Sampling}

In December 2015, March 2016, December 2016 and March 2017, 40 samples from 5 different public transport lines were collected by using a surface sampling method. Samples were taken concerning to have a high level of skin-to-touch surfaces, like grab rails and on-board stop buttons. The public transport lines were chosen concerning copy the routes with high passengers' frequency, such as proximity to Central bus or train station, proximity to Central Hospital and cemetery or students Hall of residences (Fig. 1).

The samples were collected from a predetermined area in vehicles (about $300 \mathrm{~cm}^{2}$ ) using sterile swab moistened in sterile peptone-saline solution. The samples were kept refrigerated at $4-6{ }^{\circ} \mathrm{C}$ until they were brought in the laboratory (not longer than 1 hour).

In Fig. 1, empty crosses represent proximity of the hospital, filled crosses represent proximity of cemetery and triangles represent proximity to the Hall of residence. Solid black circles represent places of sampling.

\section{Determination of S. aureus and S. aureus resistant to antibiotics}

Samples (without dilution and after their 10-fold dilution in peptone-saline solution) from each swab were plated onto Baird-Parker agar (SigmaAldrich, St. Louis, USA) in order to determine the total counts of $S$. aureus according to EN ISO 6888-1 (2003). Samples from each swab were also spread on Baird-Parker agar containing different antibiotics (ampicillin, ciprofloxacin, cefoxitin, erythromycin, gentamicin, chloramphenicol, tetracycline, vancomycin, penicillin, methicillin), all purchased from Sigma-Aldrich (St. Louis, USA). Antibiotic resistance was detected according to European (EUCAST, 2018) or for methicillin-resistance according to US (CLSI, 2007) resistance breakpoints. Before bacteriological counting, the plates were incubated for $48 \mathrm{~h}$ at $37^{\circ} \mathrm{C}$ aerobically. Each experiment was run in triplicate. The suspected colonies were confirmed by tube coagulase test, by the presence of catalase by using $3 \%(\mathrm{v} / \mathrm{v})$ solution of hydrogen peroxide, by microscopy examination and by MALDI-TOF technique.

\section{Statistical analysis}

The data analysis was performed using Microsoft Excel tools 2007 (Microsoft, Redmond, Washington, USA). Data were treated by independent Student t-test and confirmed by ANOVA test with statistical significance of $\mathrm{p}<0.05$.

\section{Results and discussion}

In all samples, the presence of $S$. aureus was confirmed. Its counts ranged from 1.40 to

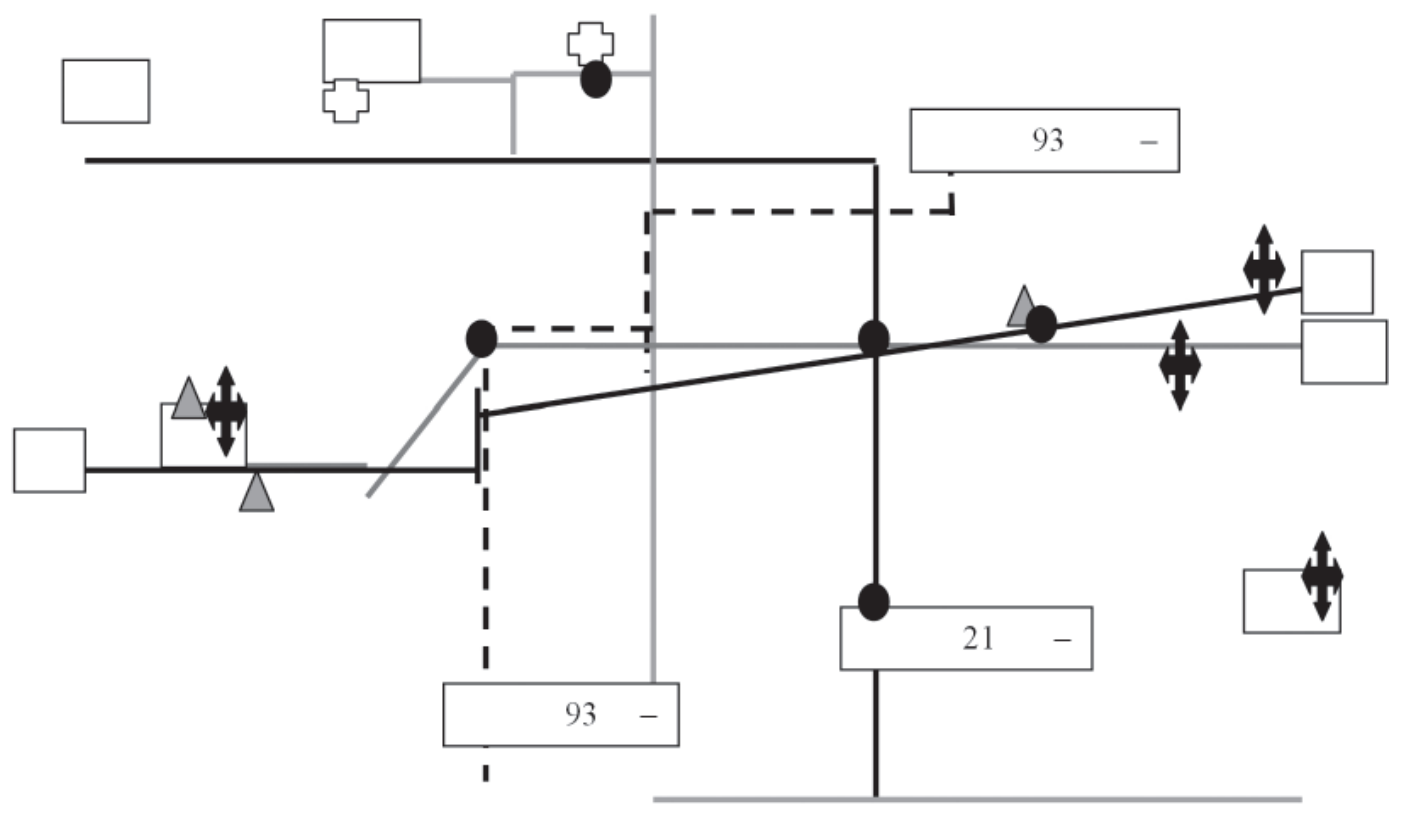

Fig. 1. Scheme of selected public transport lines in Bratislava. 


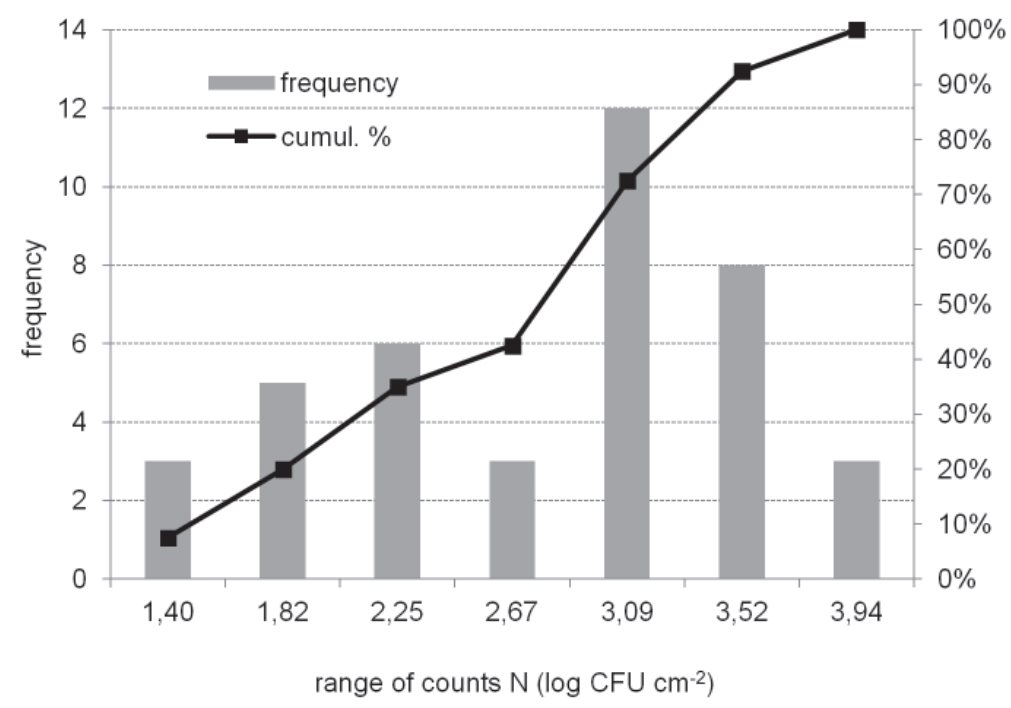

Fig. 2. The concentration of $S$. aureus contamination on hand-touched surfaces in vehicles $(n=40)$.

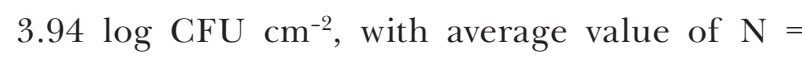
$=2.65 \pm 0.70 \log \mathrm{CFU} \mathrm{cm} \mathrm{cm}^{-2}(\% \mathrm{~V}=26.6 \%)$. The distribution of staphylococcal contamination on analysed surfaces is presented in Fig. 2. Also, Sexton et al. (2007) detected MRSA in all 2959 samples from subways, buses, trains, and other public surfaces. MRSA was also isolated from all 55 vehicles analysed by Stepanović et al. (2008). However, the proportion of samples positive for the presence of MRSA per vehicle ranged from $4.5 \%$ to $57.1 \%$. Similar results were also obtained by Otter and French (2009) who analysed the occurrence of S. aureus in buses, trains, stations, and other public places in London. They found out that $95 \%$ of analysed surfaces were colonized by $S$. aureus and its average density was about $1.08 \mathrm{CFU} \mathrm{cm}^{-2}$. On the other hand, in Lyon only $22 \%$ of analysed surfaces in buses were positive for S. aureus presence (Gaymard et al., 2016) and in Porto, only $16 \%$ of buses (mean count $6.1 \mathrm{CFU} 100 \mathrm{~cm}^{-2}$ ) and $9 \%$ of trains (mean count 4.0 CFU $100 \mathrm{~cm}^{-2}$ ) was contaminated by $S$. aureus (Mendes et al., 2015). These differences could be a result of specifically targeted sampling places close to hospitals in some studies. Moreover, as reported Mendes et al. (2015), the prevalence of $S$. aureus in public transport system in Porto decreased from $26 \%$ in 2009-2010 to $9-16 \%$ in 2015, mainly as a result of proper cleaning and disinfection practices of vehicles' surfaces.

In our samples, the amount of $S$. aureus was not significantly influenced $(\mathrm{p}<0.05)$ by the lines or by the analysed surface (grabs rails or on-board stop buttons). No significant difference between mean counts of $S$. aureus in buses and trains was also found out by Mendes et al. (2015). However, there was statistically significant $(p<0.05)$ effect of the year period, both between samples from December and March and between samples from the same month but different year.

\section{The occurrence of resistant $S$. aureus}

The occurrence of $S$. aureus resistant to 10 different antibiotics is summarized in Figure 3, where are shown all analysed samples and samples positive for the presence of $S$. aureus resistant to selected antibiotics. Only $22.5 \%$ of samples did not contain resistant $S$. aureus at all. $37.5 \%$ of samples were positive for the presence of $S$. aureus resistant to only one antibiotic, mostly to a penicillin (36.8\% of them) and methicillin (31.6\% of them). These two antibiotics, together with vancomycin were the most ineffective antibiotics in general. Altogether, $50 \%$ of samples contained $S$. aureus resistant to penicillin, and their portion in samples ranged from 67 to $99 \%$. There was also a high prevalence of resistant $S$. aureus to ampicillin (65-85\%) and cefoxitin (81-95\%). However, these isolates were detected only in 5 or 6 swabs, respectively.

The resistance to methicillin in analysed swabs ranged within 75 to $95 \%$, and $30 \%$ of swabs were positive to the presence of methicillin-resistant S. aureus. These findings are in accordance with the data presented in the Antimicrobial resistance surveillance report (ECDC, 2017). While the prevalence of MRSA strains in EU countries is continuously decreasing from $18.8 \%$ in 2012 to $16.8 \%$ in 2015 , in Slovakia a significantly increasing trend is observed. Their prevalence in 2012 was $21.7 \%$, and in 2015 it was $28.1 \%$. MRSA strains remain a public health priority not only in Slovakia but also in whole Europe, as MRSA percentages remain high (34.1 to $57.2 \%$ ) in several countries, e.g., Italy, Greece, Cyprus, 


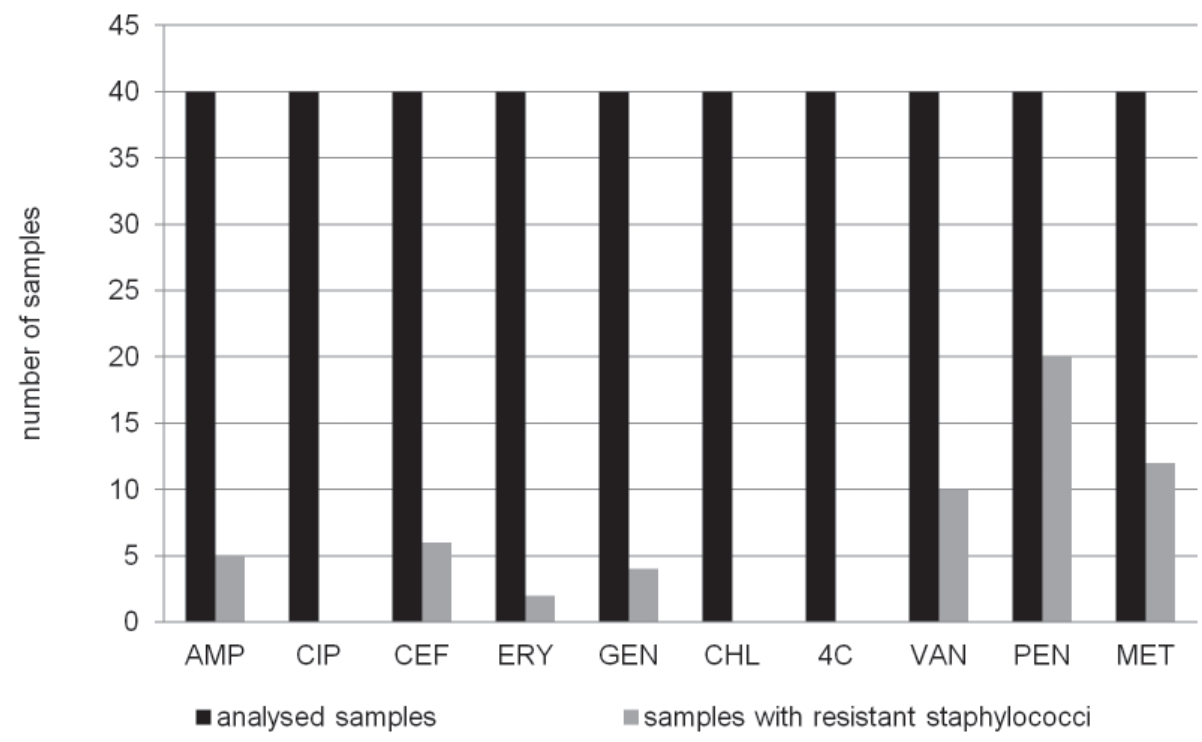

Fig. 3. The ratio of total analysed samples $(\mathrm{n}=40)$ and samples with $S$. aureus resistant against selected antibiotics. AMP - ampicillin, CIP - ciprofloxacin, CEF - cefoxitin, ERY - erythromycin,

GEN - gentamicin, CHL - chloramphenicol, 4C - tetracycline, VAN - vancomycin,

PEN - penicillin, MET - methicillin.

Portugal, Malta, and Romania. There is also a high prevalence of MRSA strains in the United States (0.2 isolates pre 1,000 patient days) with Panton-Valentine leukocidin-positive strains as a predominant compared with the United Kingdom (0.1 per 1,000) with Panton-Valentine leukocidinnegative strains as a predominant ones (Otter et al., 2009). The increased MRSA infections reported in different countries led to the detection of unique SCCmec element, that appears more genetically mobile, and it is suggested that it has been heterologously transferred from other staphylococcal species (Lowy, 2014). MRSA isolates are often also resistant to fluoroquinolones, further limiting the treatment options available for severe infections. In this context, it was surprising, that there was no ciprofloxacin-resistant $S$. aureus in our samples.

Furthermore, there was also no $S$. aureus resistant to chloramphenicol and tetracycline. Contrary results obtained by Stepanović et al. (2005) indicating a high prevalence of coagulase-negative staphylococci resistant to tetracycline $(63.1 \%)$, followed by gentamicin (33.7 \%) and erythromycin $(33 \%)$ resistant strains. From vehicles moving around Bratislava, S. aureus resistant to gentamicin and erythromycin were confirmed in 10 or $5 \%$ of swabs, respectively. Stepanović et al. (2005) also found out that $46.9 \%$ of staphylococci detected in Belgrade's public transport were multidrugresistant. In our case, only $15 \%$ of isolates can be considered as multidrug-resistant $S$. aureus according to criteria proposed by Magiorakos et al. (2011).

\section{Conclusion}

In conclusion, we found out that $S$. aureus was detected in all samples from public transport surfaces. Only $23 \%$ of samples did not contain S. aureus resistant to some of 10 analysed antibiotics. On the other hand, the severe prevalence of highly resistant $S$. aureus to penicillin, methicillin, ampicillin, and cefoxitin was confirmed. So, public surfaces can be a significant reservoir not only for $S$. aureus but also for its resistant variants.

Further work is required to assess the prevalence of S. aureus also in other Slovak and European cities and also to assess the transfer of resistant bacteria from the community to environment, and vice versa. Also, the appropriate cleaning and disinfection practices are needed in order to reduce the prevalence of pathogenic bacteria in the environment together with appropriate personal hygiene practices. Last (but) not least, the well-considered and reasonable usage of antibiotics is a global necessity.

\section{Acknowledgment}

This work was supported by the Slovak Research and Development Agency (Contract No. APVV-16-0171) and by the Scientific Grant Agency of the Ministry of Education of the Slovak Republic and Slovak Academy of Science - VEGA 1/0096/17.

\section{References}

Arbuthnott J, Coleman D, DeAzavedo J (1990) Journal of Applied Bacteriology Symposium Supplement 69: 1S-8S. 
Bremer P, Fletcher G, Osborne C (2004) Staphylococcus aureus, New Zealand Institute for Crop and Food Research Limited, Christchurch. pp. 1-6.

CLSI Performance Standards for Antimicrobial Susceptibility Testing (2007) $17^{\text {th }}$ Informational Supplement 27: 1-182.

ECDC (2017) Antimicrobial resistance surveillance in Europe 2015, Surveillance report, Avialable in May 2017: http://ecdc.europa.eu/en/publications/ Publications/antimicrobial-resistance-europe-2015.

EN ISO 6888-1 (2003) Microbiology of food and animal feeding stuffs - Horizontal method for the enumeration of coagulase-positive staphylococci (Staphylococcus aureus and other species) - Part 1: Technique using Baird-Parker agar medium, 1-11.

European Committee on Antimicrobial Susceptibility Testing (EUCAST, 2018) Breakpoint tables for interpretation of MICs and zone diameters. Version 7.1., Available in July 2018: http//www.eucast.org.

Gaymard A, Pichon M, Degaud M, Tasse J, Dupieux C, Laurent F (2016) International Journal of Antimicrobial Agents 48: 459-462.

Lowy FD (2014) Journal of Clinical Investigation 111: $1265-1273$.
Magiorakos A-P, Srinivasan A, Carey RB, Carmeli Y, Falagas ME, Giske CG et al. (2011) Clinical Microbiology and Infection 18: 268-281.

Mendes Â, Martins da Costa P, Rego D, Beça N, Alves C, Moreira T, Conceiçao T, Aires-de-Sousa M (2015) Public Health 129: 1125-1131.

Mylotte JM, McDemott C, Spooner JA (1987) Reviews of Infectious Diseases 9: 891-907.

Otter JA, French GL (2009) Letters in Applied Microbiology 49: 803-805.

Otter JA, Havill NL, Boyce JM, French GL (2009) Journal of Clinical Microbiology and Infectious Diseases 28: 835-839.

Reffuveille F, Josse J, Vallé Q, Mongaret C, Gangloff SC (2017) In Enany S, Crotty Alexander LE (Eds.) The rise of virulence and antibiotic resistance in Staphylococcus aureus, (pp. 189-214). InTech, Zagreb.

Sexton J, Maxwell S, Gerba S (2007) 107 ${ }^{\text {th }}$ Annual Meeting of the American Society for Microbiology.

Stepanović S, Ćirković I, Djukić S, Vuković D, ŠvabićVlahović M (2008) Letters in Applied Microbiology 47: 339-341. 